\title{
Internal pressure and façade loads on a residential tall building obtained from wind tunnel tests
}

\author{
S. Giappino, L. Rosa, G. Tomasini \& A. Zasso \\ Dipartimento di Meccanica, Politecnico di Milano, Italy
}

\begin{abstract}
Wind tunnel studies on a residential tall building and a numerical model to evaluate the façade design wind loads are presented. External and internal pressure measurements are carried out on a rigid model in a geometric scale factor equal to $1 / 100$. The pressure inside the building is investigated in a top corner "room" as a significant test condition. The case with a single dominant opening is studied as it is considered the worst configuration. Tests show that, according to previous studies on low-rise buildings, the internal pressure closely follows the external pressure at the opening. The experimental data obtained from the "typical room" are used to calibrate a numerical model adopted to calculate the net-pressure coefficients on the entire surface of the tower.

Keywords: wind loads, wind tunnel test, internal pressure, net pressure, external pressure, net-load numerical model, tall building, single opening.
\end{abstract}

\section{Introduction}

Turbulence of the incoming wind and flow pattern around a building generate a fluctuating external pressure field on the building itself. The internal pressure could be dependent on this external pressure field. For sealed buildings, the internal pressure is small compared with the external pressure. However, in some circumstances the internal pressure can assume large values. On tall buildings an important design case for a cladding at a corner could be a wall opening at the adjacent wall at the same corner, maybe caused by a window left open during a strong storm. The combination of a large internal pressure and a external suction (and vice-versa) can generate a large net-load across the envelope and cause the failure of a cladding. Therefore this is an important design load condition. 
It has long been wind engineering practice to study external pressure by using wind tunnel tests while internal pressure is more difficult to capture accurately, because it is dependent on many factors: position and size of the openings, internal volume, porosity of the surface and envelope flexibility. For this reason the quasisteady design approach indicated by many wind loading standards (for instance AS1170.2 [1] or EuroCode1 [2]) is usually used. However recent studies on lowrise buildings (for instance Ginger and Letchford [3]) have shown that the internal pressure in the case of a building with a dominant opening has a strong dynamic component and the net-pressures derived are greater than the values obtained by the codes.

This work presents the studies carried out on a residential tall building which is $132 \mathrm{~m}$ high, Fig. 1(a), in order to evaluate the façade design wind loads. As the tower is residential, it is expected that there will be different possible openings, also dominant ones. Starting from the experimental values of the internal pressure obtained in a box representative of a "typical room" of the building, a numerical model is calibrate and adopted to estimate the net-load on the overall surface of the tower.

\section{Experimental details}

The experiments are performed on a rigid model of the building in a geometric scale factor equal to 1/100, Fig. 1(a), in the Boundary Layer Test Section of the Wind Tunnel of the Politecnico di Milano, that is $4 \mathrm{~m}$ high, $14 \mathrm{~m}$ wide and $36 \mathrm{~m}$ long. Pressure measurements are carried out in condition of atmospheric boundary layer simulation. The turbulent atmospheric boundary layer simulation is achieved by using passive turbulence generators, spires and roughness elements, placed in the wind tunnel test section entrance. The mean velocity profile and vertical turbulent intensity profile used during the tests are representative of a urban terrain category.

The internal pressures in buildings with more openings is not the same into all the internal volumes. In the wind tunnel tests carried out for this study, one single dominant opening is considered for a top corner apartment, hereafter referred as "typical room". One single dominant opening is considered because this is the worst situation:in fact if there are more openings, the internal pressure will be a mean of the external pressures, and as a consequence, the net-load will be lower, [3]. This "typical room" consists of a box that is placed on the top corner of the tower and is isolated from the other parts of the building. It has a single dominant opening, exactly in correspondence of an external pressure tap hereafter called tap 0925, Fig. 1(b) and Fig. 2. Inside this box three pressure taps (named APPA1, APPA2 and APPA3) are placed. The pressure measurements are performed using the high-speed scanning pressure equipment PSI-system 8400. The small dimensions of the scanners allow to place them into the model close to the measuring points, reducing the length of the tubes. Data acquisition is made at a sampling rate of $100 \mathrm{~Hz}$ for a period of $100 \mathrm{~s}$, corresponding to more than 1 hour full scale. The pressure on the external surface of the tower are defined by measurements in a 


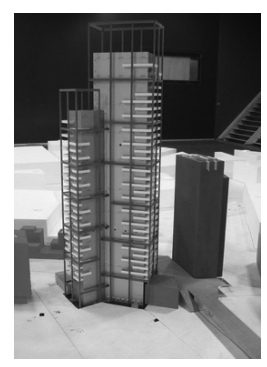

(a) The entire model tested.

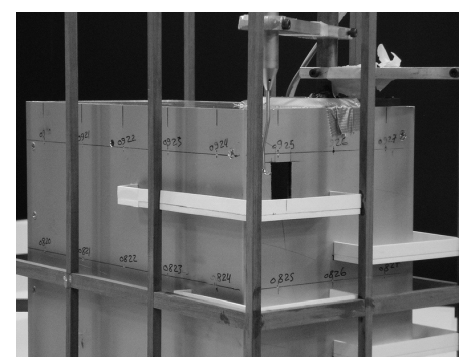

(b) The opening at the top corner of the tower.

Figure 1: The wind tunnel test configuration on the rigid model.

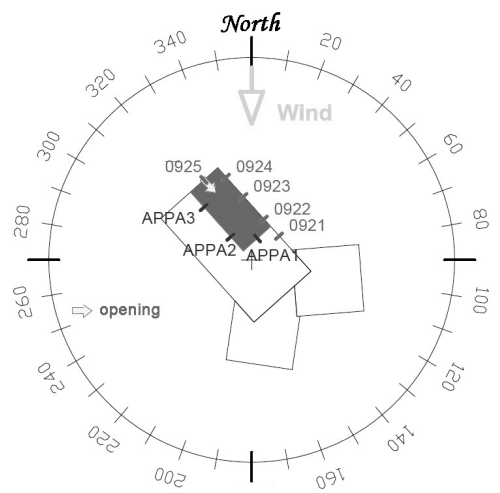

Figure 2: Top view of the building: position of the "typical room"at the top of the tower and of the pressure taps around and inside the box.

total of 224 discrete points distributed on the model. The internal and external pressures are acquired simultaneously, with and without the presence of the opening. The results are calculated in dimensionless form as local pressure coefficients as a function of the wind exposure angle, $C_{P}(\alpha)$. The maximum peak value $\hat{C}_{P}(\alpha)$ and the minimum peak value $\check{C}_{P}(\alpha)$ are calculated using the Cook and Mayne method [4].

\section{Experimental results on the "typical room"}

\subsection{Internal and external experimental pressure coefficients}

Figure 3(a) shows the mean value $\bar{C}_{\text {Pint }}$ (square, $\square$ ), the maximum peak value $\hat{C}_{\text {Pint }}$ (triangle up, $\mathbf{\Delta}$ ) and the minimum peak value $\breve{C}_{\text {Pint }}$ (triangle down, $\boldsymbol{\nabla}$ ), of the 


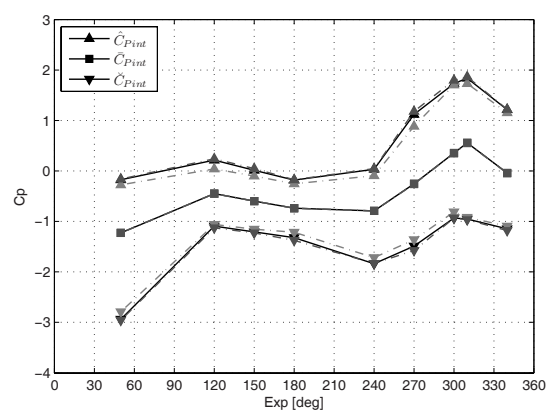

(a)

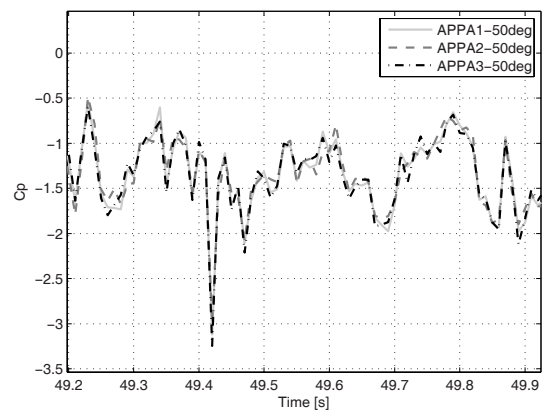

(b)

Figure 3: Internal pressure coefficients comparison: (a) mean and peak values APPA1, APPA 2 and APPA3 vs wind direction $\alpha$; (b) time-histories zoom $\alpha=50 \mathrm{deg}$.

Table 1: Normalize cross-correlation between the internal pressure taps.

\begin{tabular}{|c|c|c|}
\hline xcov & $50 \mathrm{deg}$ & $310 \mathrm{deg}$ \\
\hline APPA1-APPA2 & 0.965 & 0.963 \\
APPA1-APPA3 & 0.977 & 0.978 \\
APPA2-APPA3 & 0.938 & 0.940 \\
\hline
\end{tabular}

internal pressure coefficients (taps APPA1-2-3) as a function of the incoming wind direction $\alpha$. Figure 3(b) shows an interval of the time histories of these coefficients for the exposure angle $\alpha=50 \mathrm{deg}$. Table 1 shows the normalize cross-correlation between the time histories of the internal pressures for the exposition angles $\alpha=$ $50 \mathrm{deg}$ and $\alpha=310 \mathrm{deg}$. The analysis of the data shows that the mean, peak and instantaneous values of the pressure coefficients measured by the three considered taps, are very close and the normalize cross-correlations are close to one, then the pressure inside an internal volume with one single dominant opening can be assumed as homogeneous. In the next, the pressure acquired by tap APPA2 is assumed as the internal pressure inside the "typical room".

Figure 4 shows the external pressure coefficient $C_{\text {Pext }}$ measured by two taps located outside the "typical room"between the sharp edge of the two perpendicular sidewalls, as shown in Fig. 2, as a function of the exposition angle $\alpha$. The values in the figure are acquired with the presence of the opening; however, the comparison with the values of the same pressure taps obtained without the opening shows that their trend is totally similar. In Fig. 5 the external pressure coefficient $C_{\text {Pext,0925 }}(\alpha)$ of the pressure tap located close to the opening is plotted along with $C_{\text {Pint }}(\alpha)$. It 


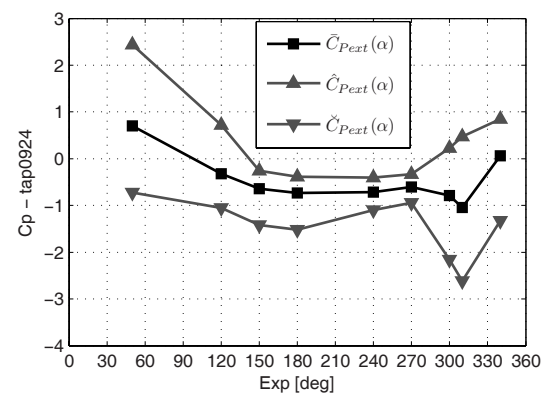

(a)

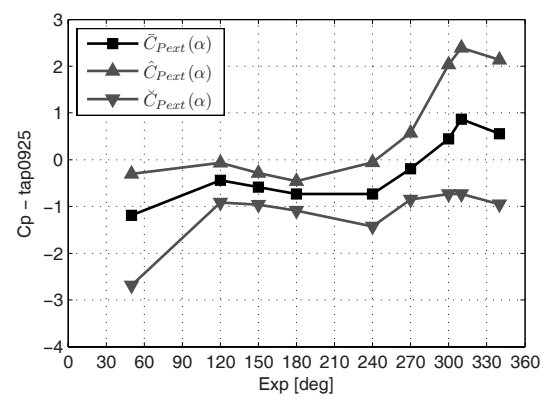

(b)

Figure 4: External pressure coefficients vs wind angle $\alpha$ of the taps 0924 (a) and 0925 (b).

is possible to see very clear that both the mean and the peak values are very close, being the only appreciable distinction between the positive peaks at $310 \mathrm{deg}$. In Fig. 6 an interval of the time histories of these signal are plotted. Two exposition angles are considered: $\alpha=50 \mathrm{deg}$ and $\alpha=310 \mathrm{deg}$, that are where the minimum and maximum of the peak and mean values are recorded. Their standard deviation and normalize cross-correlation values are reported in Table 2.

The analysis shows that the pressure inside the "typical room" follows closely with the external pressure close to the opening (tap 0925), mainly when the pressure is negative ( $\alpha=50 \mathrm{deg}$, Fig. 6(a)). In correspondence of the wind exposures where the mean pressure value is positive, the internal mean value is a bit lower, but the dynamic component, as one can see from the standard deviation and the normalize cross-correlation, is the same ( $\alpha=310 \mathrm{deg}$, Table 2$)$. This behaviour is connected with the mass of air that flows in and out of the building and the consequent changing of the density; a detail treatment could be found for instance in [5]. So, when a building is enveloped by turbulent flow, the external pressure has significant fluctuations, and, if a dominant opening is present, the internal pressure shows the same dynamics: the pressure inside the flat responds quickly and follows completely in phase the changes of the external pressure close to the dominant opening.

\subsection{Experimental net-pressure coefficient on the façade}

The net-pressure (external-internal) coefficient on the external surface of the "typical room" is evaluated as:

$$
C_{P n e t, m}(t, \alpha)=C_{P, m}(t, \alpha)-C_{P i n t}(t, \alpha)
$$




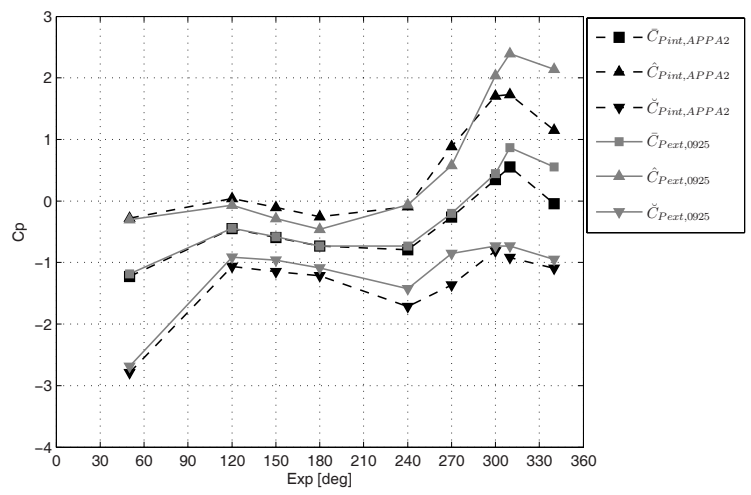

Figure 5: Pressure coefficients vs exposure angle $\alpha$ : comparison between internal tap APPA2 and external tap 0925 in terms of mean value $\bar{C}_{P}(\alpha)$ and peak values $\hat{C}_{P}(\alpha)$ and $\breve{C}_{P}(\alpha)$.

Table 2: Standard deviation and normalize cross-correlation. Internal and external (tap 0925) pressure coefficient.

\begin{tabular}{|c|c|c|c|}
\hline Exposure angle & $\sigma_{A P P A 2}$ & $\sigma_{0925}$ & $x_{\text {cov }_{A P P A 2}-0925}$ \\
\hline $50 \mathrm{deg}$ & 0.28 & 0.26 & 0.795 \\
$310 \mathrm{deg}$ & 0.30 & 0.29 & 0.727 \\
\hline
\end{tabular}

where $C_{P, m}$ is the pressure coefficient evaluate in the $m$-point outside the "typical room" (taps 0922-3-4-5, Fig. 1(b)), and $C_{\text {Pint }}$ is the internal pressure coefficient (tap APPA2). So a positive value of the coefficient $C_{P n e t}$ means a net-positive load on the surface, while a negative value of the same coefficient means a suction load on the surface. Figure 7 shows the net-pressure coefficients $\bar{C}_{\text {Pnet }}(\alpha), \hat{C}_{\text {Pnet }}(\alpha)$ and $\breve{C}_{\text {Pnet }}(\alpha)$, calculated, for each wind exposure, right from the coefficient $C_{\text {Pnet }}(t)$ obtained by eq. (1). The comparison with Fig. 4 shows that generally the load on the wall adjacent the surface with the opening is greatly increased by the presence of a dominant opening, 7(a). Besides, the net-load on the wall with the opening (tap 0925), is lower, Fig. 7(b). The most critical configurations occur in the exposition angles $\alpha=50 \mathrm{deg}$ and $\alpha=310 \mathrm{deg}$, that is when the wind is perpendicular, or alongside, to the wall with the opening. The strongest positive net-load occur in the exposition $\alpha=50 \mathrm{deg}$, when the wind is alongside the wall with the opening. The internal pressure, that follows the external pressure close to the opening, reaches its minimum, while the external pressure on the sidewall shows its maximum peak value. The highest positive external pressure is so combined with the lowest negative internal pressure, resulting in a strong positive net-load on the surface of the sidewall. 


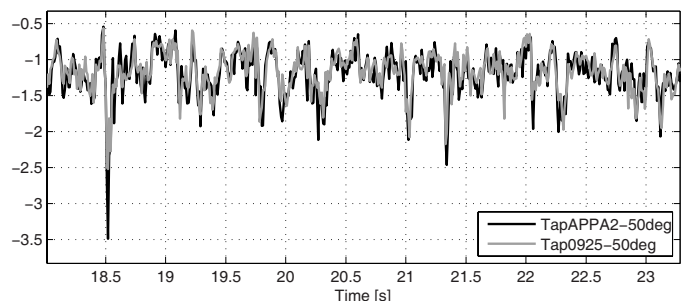

(a)

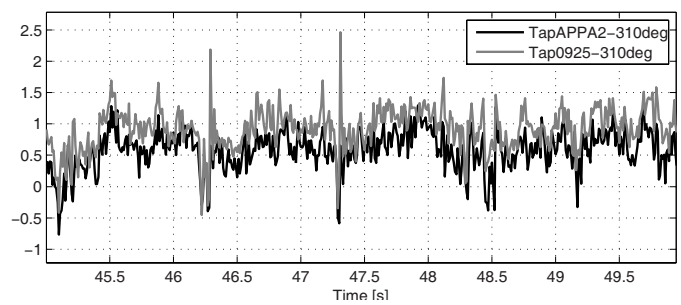

(b)

Figure 6: A detail of the time histories of taps APPA2 and 0925. (a) $C_{\text {Pint }}(t)$ and $C_{\text {Pext }, 0925}(t) . \alpha=50 \mathrm{deg}$. Negative mean value. (b) $C_{\text {Pint }}(t)$ and $C_{\text {Pext }, 0925}(t) . \alpha=310 \mathrm{deg}$. Positive mean value.

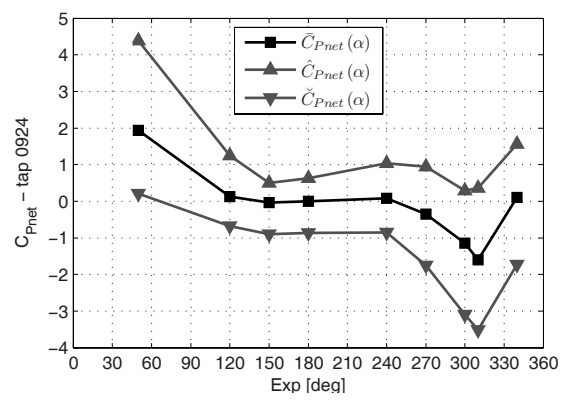

(a)

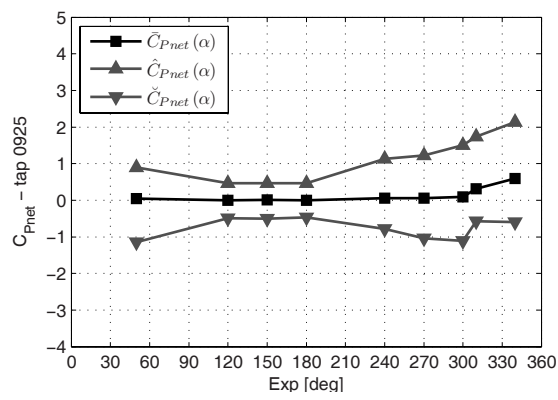

(b)

Figure 7: Net-pressure coefficient vs. exposure angle: $\bar{C}_{\text {Pnet }}(\alpha), \hat{C}_{\text {Pnet }}(\alpha)$ and $\breve{C}_{\text {Pnet }}(\alpha)$ for the tap 0924 (a) and the tap 0925 (b). 


\section{Model for the evaluation of the net-load on all the surface of the tower}

\subsection{Model for the evaluation of the net-pressure coefficient}

In order to evaluate the net-load in correspondence of all the external pressure taps, a model has been set up starting from the results obtained for the "typical room". The resulting net-pressure coefficients at each external pressure taps $n, \bar{C}_{P n e t, n}$, $\hat{C}_{\text {Pnet }, n}$, and $\breve{C}_{\text {Pnet }, n}$, can be calculated, for each exposure angle, with the following expressions:

$$
\begin{aligned}
& \bar{C}_{\text {Pnet }, n}=\bar{C}_{\text {Pext }, n}-\bar{C}_{\text {Pint }} \\
& \hat{C}_{\text {Pnet }, n}=k_{1} \cdot \hat{C}_{\text {Pext }, n}-k_{2} \cdot \breve{C}_{\text {Pint }}^{\approx} \\
& \breve{C}_{\text {Pnet }, n}=k_{3} \cdot \breve{C}_{\text {Pext }, n}-k_{4} \cdot \hat{C}_{\text {Pint }}^{\approx}
\end{aligned}
$$

where $\bar{C}_{\text {Pext }, n}, \hat{C}_{\text {Pext }, n}$ and $\breve{C}_{\text {Pext }, n}$ are the mean value and the peak maximum and minimum of the external pressure at tap $n$ respectively, while $\bar{C} \approx{ }_{\text {Pint }}, \hat{C}_{\text {Pint }}$ and $\breve{C}_{\text {Pint }}$. are an estimation of, respectively, the mean value and the peak maximum and minimum of the of the internal pressure. $k_{i}$ are constants evaluated by the experimental tests.

\subsection{Estimation of the internal pressure coefficient}

The tests carried out on the "typical room" at the top of the tower have shown that the internal pressure follows quite well the external pressure close to the dominant opening. As consequence, the internal pressure coefficient can be evaluated right from the external pressure coefficient, also when the measurement of $C_{\text {Pint }}(t)$ is not available.

Each floor is assumed to be an isolated internal volume (floor is intended as pressure taps level). The computation is achieved for each $j$-floor and for each $n$-tap. Then, looking at the external pressures at that $j$-level, as the $C_{n e t}$ must be used for design purpose, the worst situation is considered, that is:

- if $\bar{C}_{\text {Pext }, n}^{j}>0 \rightarrow \bar{C}_{\text {Pint }}^{j, \approx}=$ the most negative external mean coefficient on the $j$-floor.

- if $\bar{C}_{\text {Pext }, n}^{j}<0 \rightarrow \bar{C}_{\text {Pint }}^{j, \approx}=$ the most positive external mean coefficient on the $j$-floor. If the most positive external mean coefficient on the floor is negative, then it is assumed $C_{\text {Pint }}^{\approx}=0$.

- positive peak values, $\hat{C}_{\text {Pnet }, n}^{j} \rightarrow \breve{C}_{\text {Pint }}^{j, \approx}=$ the most negative external minimum peak coefficient of the $j$-floor.

- negative peak values, $\breve{C}_{\text {Pnet }, n}^{j} \rightarrow \hat{C}_{\text {Pint }}^{j, \approx}=$ the most positive external maximum peak coefficient of the $j$-floor.

This is a safer method since for each wind exposure angle it assumes the opening where the maximum (or minimum) pressure on the considered $j$-floor occurs. 


\subsection{Estimation of the factors $k_{i}$}

Since the peak values of external pressure coefficients, $\hat{C}_{P e x t, n}$ and $\breve{C}_{\text {Pext, } n}$, and the peak values of the estimated internal pressure coefficient, $\hat{C_{\text {Pint }}}$ and $\breve{C} \widetilde{\text { Pint }}$, are not expected to be simultaneous, it is necessary to use a proportional factor, $k_{i}$, in the calculation of $\hat{C}_{\text {Pnet }, n}$ and $\breve{C}_{\text {Pnet }, n}$. The results given by the wind tunnel tests on the "typical room" are also used to calibrate these parameters.

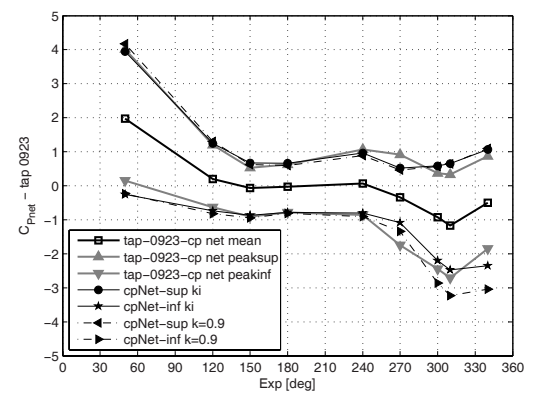

(a)

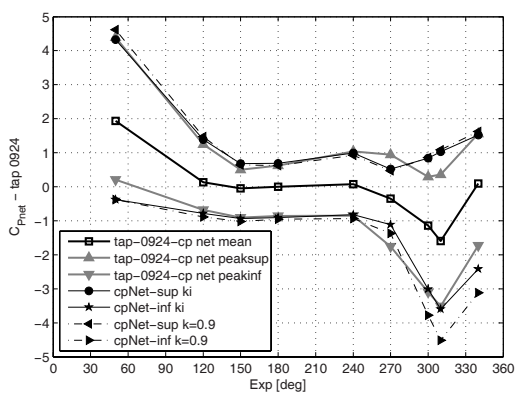

(b)

Figure 8: Net-pressure coefficient estimated and experimentally measured vs exposure angle: $\bar{C}_{\text {Pnet }}(\alpha), \hat{C}_{\text {Pnet }}(\alpha)$ and $\breve{C}_{\text {Pnet }}(\alpha)$. (a) tap 0923, (b) tap 0924.

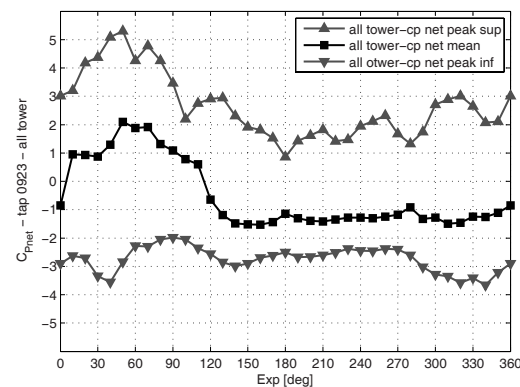

(a)

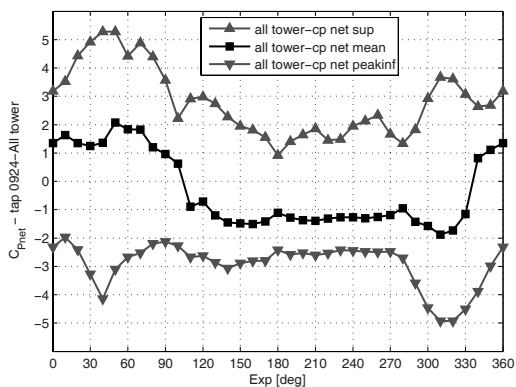

(b)

Figure 9: Net-pressure coefficient estimated considering all the pressure taps on that level: $\bar{C}_{\text {Pnet }}^{\dagger}(\alpha), \hat{C}_{\text {Pnet }}^{\dagger}(\alpha)$ and $\breve{C}_{\text {Pnet }}^{\dagger}(\alpha)$. (a) tap 0923, (b) tap 0924. 
The method considers the following equality:

$$
\begin{aligned}
& \hat{C}_{\text {Pnet }, m}^{E X P}=k_{1} \cdot \hat{C}_{\text {Pext }, m}^{E X P}-k_{2} \cdot \breve{C}_{\text {Pint }, \approx}^{E X P} \\
& \breve{C}_{\text {Pnet }, m}^{E X P}=k_{3} \cdot \breve{C}_{\text {Pext }, m}^{E X P}-k_{4} \cdot \hat{C}_{\text {Pint }}^{E X P}
\end{aligned}
$$

where the apex EXP means experimental data, $m$ refers to the taps considered in the analysis (that are the taps 0922-3-4) and the internal pressure is assumed to be equal to the external pressure close to the wall opening:

$$
C_{\text {Pint }}^{E X P} \approx=C_{P, 0925}^{E X P}
$$

Through an optimization process, carried out considering the $m$-taps and all the exposure angles, the following values of $k_{i}$ have been estimated: $k_{1}=0.77, k_{2}=$ $0.91, k_{3}=0.79, k_{4}=0.64$. All the $k_{i}$ values are similar, meaning that all the pressure coefficients have a similar influence on the calculation of the net-load on the cladding. For this reason and for simplicity, one single value of $k$ may be considered, which is chosen as $k=0.9$ for safety reasons. Figure 8 shows the netpressure coefficients outside the "typical room" measured experimentally (eq. (1) and Fig. 7) plotted along with the net-coefficients estimated by the optimization method, eq. (3), both using different values of $k_{i}$ and using $k=0.9$ for all the $k_{i}$. One can see that the values of the experimental and the calculated $C_{P n e t}$ are in good agreement and that the use of $k=0.9$ obtains safer results. Lastly, Fig. 9 shows the net-pressure coefficients of the taps 0923 and 0924 evaluated according to the model proposed by eq. (2) considering, in the calculation, all the pressure taps on that floor. As previously observed, the model is a safer tool and the values are much greater than those obtained directly from the external pressure (Fig. 4).

The net-pressure design load on the façade can finally be calculated as:

$$
P_{n}(\alpha)=\frac{1}{2} \rho V^{2}(\alpha) \cdot C_{P n e t, n}(\alpha)
$$

where $\rho$ is the density of the air, $V(\alpha)$ is the mean design wind speed and $C_{\text {Pnet, } n}(\alpha)$ is taken as $\hat{C}_{\text {Pnet }, n}(\alpha)$ or $\breve{C}_{\text {Pnet }, n}(\alpha)$.

\section{Conclusion}

Wind tunnel tests on a rigid scale model of a residential tall building have been made to determine the net-load (external-internal) on the building envelope.

Since the tower is residential, it is expected there will be different possible openings, also dominant ones. This condition may generate large internal pressures in strong wind conditions, therefore it is necessary to consider not only the pressure acting on the external surfaces, but also the internal ones. In order to evaluate the internal pressure a box with a dominant wall opening, representative of a "typical room" of the tower, has been made on the top of building.

The tests have shown that the internal pressure is homogeneous inside the "typical room" and its mean and fluctuating value closely follows the external pressure 
close to the opening. The presence of a dominant wall opening reduces the net load on that wall, but greatly increases the net load on the sidewalls. The worst configuration occurs when the wind is perpendicular or parallel to the wall opening, since the internal pressure shows its maximum, or minimum, value.

An original method to predict the net-load without having the measurements of the internal pressure has been set up and calibrated through the results obtained on the "typical room". This calculation method is based on some assumptions: the internal pressure follows closely the external pressure at the wall opening (this has been verified experimentally), each floor is assumed an isolated volume, the wall opening is located in the worst position on the floor. The comparison between the experimental coefficients and the values obtained from the method is good. The net-loads calculated with this method, show values much greater than that obtained considering only the external pressures. This shows the importance of considering also the internal pressure in the design stage of the façade.

\section{Acknowledgement}

The Authors wish to acknowledge the support of Dr. Andrew Allsop for his helping in the discussion of the results.

\section{References}

[1] AS-1170.2, Australian standard saa loading code part 2, wind loads. Technical report, 1989.

[2] UNI EN 1991-1-4, Eurocodice1. Azioni sulle strutture. Parte 1-4: Azioni in generale - Azioni del vento. Eurocode, 2005.

[3] Ginger, J.D. \& Letchford, C.W., Net pressures on a low-rise full-scale building. Journal of Wind Engineering and Industrial Aerodynamics, 83, pp. 239250, 1999.

[4] Cook, N.J., The designer's guide to wind loading of building structures. Building Research Establishment report, Building Research Establishment, Dept. of the Environment; Butterworths: [Garston, Watford]; London; Boston, 1985.

[5] Holmes, J.D., Wind loading of structures. Spon Press; Taylor \& Francis, 2004. 